\title{
Noise Filtering to Improve Data and Model Quality for Crowdsourcing
}

\author{
Chaoqun Li ${ }^{\mathrm{a}}$, Victor S. Sheng ${ }^{\mathrm{b}}$, Liangxiao Jiang ${ }^{*, \mathrm{c}}$, Hongwei $\mathrm{Li}^{*}$,a \\ ${ }^{a}$ Department of Mathematics, China University of Geosciences, Wuhan 430074, China \\ ${ }^{b}$ Department of Computer Science, University of Central Arkansas, Conway, AR, USA \\ ${ }^{c}$ Department of Computer Science, China University of Geosciences, Wuhan 430074, China
}

\begin{abstract}
Crowdsourcing services provide an easy means of acquiring labeled training data for supervised learning. However, the labels provided by a single crowd worker are often unreliable. Repeated labeling can be used to solve this problem. After multiple labels have been acquired by repeated labeling for each instance, in general consensus methods are used to obtain the integrated labels of instances. Although consensus methods are effective in practice, it cannot be denied that a level of noise still exists in the set of integrated labels. In this study, an attempt was made to employ noise filters to delete the noise in integrated labels, and consequently, enhance the training data and model quality. In fact, noise handling is a relatively mature field in the machine learning community, and many noise filters for deleting label noise have been presented in the past. However, to the best of our knowledge, in very few studies was noise filtering used to improve crowdsourcing learning. Therefore, in this study we empirically investigated the performance of noise filters in terms of improving crowdsourcing learning. Thus, in this paper some existing noise filters presented in previous papers are reviewed and their experimental application to crowdsourcing learning tasks is described. Experimental results based on 14 benchmark UCI data sets and three real-world data sets show that these noise filters can significantly reduce the noise level in integrated labels and thereby considerably enhance the performance of target classifiers.
\end{abstract}

Key words: Crowdsourcing learning; Integrated labels; Label noise; Noise filtering.

\section{Introduction}

In supervised learning, a training instance is always denoted ${ }^{26}$ by a $d$-dimensional feature vector and a known label. The traditional technique for acquiring the known label from domain ${ }_{29}$ experts is expensive and time-consuming in many cases. With ${ }_{30}$ the development of the Internet techniques, crowdsourcing services, such as Amazon Mechanical Turk, have become an efficient and low cost means of obtaining a great quantity of labeled data for supervised learning. However, some factors, such as the low payment of crowd workers and their limited ${ }_{35}$ abilities, lead to the labels provided by a single crowd worker ${ }_{36}$ frequently being unreliable. To solve this problem, multiple ${ }_{37}$ labels are frequently requested from different crowd workers for an instance, that is, repeated labeling is performed. Sheng et al. (2008) proved that, when a single worker's labeling is not ${ }_{40}$ perfect, repeated labeling is an effective approach to improv- ${ }_{41}$ ing the quality of integrated labels. After acquiring multiple ${ }_{42}$ labels of an instance by repeated labeling, consensus methods, such as Majority Voting (MV), RY (Raykar et al., 2010), and ZenCrowd (ZC) (Demartini et al., 2012), can be used to infer a ${ }^{44}$ training label (an integrated label, i.e., a best consensus label) ${ }^{45}$ of the instance. A relatively comprehensive review of consensus methods can be found in (Sheshadri \& Lease, 2013; Guo ${ }_{47}$ et al., 2014).

\footnotetext{
*Corresponding authors: Liangxiao Jiang, Hongwei Li

Email addresses: $1 \mathrm{j}$ iang@cug. edu . cn (Liangxiao Jiang),
} hwli@cug.edu.cn (Hongwei Li) ists in a set of integrated labels inferred by consensus methods. Here, noise refers to the instances, the integrated labels of which are different from their true labels, i.e., the labels given by domain experts. For example, after multiple labels of an instance are acquired from different crowd workers, MV infers the integrated label by majority voting. MV is the simplest consensus method and runs fast, but its simplicity may come at the price of low integrated label quality. More complicated consensus methods may result in better integrated label quality than MV. However, noise is still inevitably present in the integrated labels.

Here, we use an illustrative example to show the noise level in integrated labels. For the discussion below, we give some notations and definitions. $D=\left\{\left(\mathbf{x}_{i}, y_{i}\right)\right\}_{i=1}^{M}$ is a training data set containing $M$ instances, where $\mathbf{x}_{i}$ is the instance described by a $d$-dimensional feature vector and $y_{i}$ is the corresponding known true label. $U=\left\{u_{j}\right\}_{j=1}^{R}$ denotes the labelers of a crowdsourcing system. Each instance $\mathbf{x}_{i}$ has a multiple label set $\mathbf{l}_{i}=\left\{l_{i j}\right\}_{j=1}^{R}$, where $l_{i j}$ is the label of the instance $\mathbf{x}_{i}$ annotated by the labeler $u_{j}$

Definition 1. (Overall labeling quality). The overall labeling quality of a labeler $u_{j}$ is the probability that the label of an instance $\mathbf{x}_{i}$ annotated by the labeler $u_{j}$ is the true label, notated as $p_{j}$

Definition 2. (Integrated label quality). After acquiring the multiple label set of an instance $\mathbf{x}_{i}, \mathbf{l}_{i}=\left\{l_{i j}\right\}_{j=1}^{R}$, a certain con- 
sensus method is used to induce its integrated label, notated as $\hat{y}_{i}$; the integrated label quality is the probability that the integrated label is the true label, notated as $q_{i}$.

Definition 3. (Noise ratio). The noise ratio (NR) of a set of integrated labels of a data set is the percentage of instances, the integrated labels of which are different from their true labels. The NR can be calculated as $N R=\sum_{i=1}^{M} I\left(\hat{y}_{i} \neq y_{i}\right) / M$, where $I(\bullet)$ is a binary function, which is 1 when this condition in this bracket is met; otherwise, 0 .

In order to show the noise level in integrated labels clearly, we designed two simulation experiments. We randomly select a data set breast-cancer (see Section 4), and the true labels of all instances are hidden. The first experiment employs nine labelers, and each labeler generates a label for every instance according to a labeling quality $p_{j}$ : the true label of every instance is assigned to the instance with probability $p_{j}$ and the opposite value is assigned with probability $1-p_{j}$. Here, we consider a simple case where the labeling quality of all labelers is the same, that is, $p_{j}=p$ for all $j$ (we relax this assumption in Section 4). After obtaining nine labels for every instance, consensus methods MV, RY, and ZC are used to infer the integrated label of every instance. Notice that, according to a previous study (Sheng et al., 2008), $p>0.5$ must be satisfied; otherwise, the integrated label quality $q_{i}$ cannot be improved by repeated labeling. The second experiment employs different numbers of labelers, but the labeling quality $p$ is fixed $(p=0.6)$ and MV, $\mathrm{RY}$, and ZC are used to infer the integrated label of every instance.

Figure 1 shows the reduction in the NR in the set of integrated labels with the increase in labeling quality. Figure 2 shows the reduction in NR with the increase in the number of labelers. In Figure 1, we can see that, when the labeling quality is relatively high, the NR is relatively low, and when the labeling quality $p$ approaches 0.9 , the NR approaches 0 . This means that when ${ }^{108}$ the labeling quality is quite high there is almost no noise in the ${ }^{109}$ set of integrated labels. Unfortunately, in real-world applica- ${ }^{110}$ tions, the labeling quality is frequently low. In Figure 2 we can ${ }^{111}$ see that, when the labeling quality of each labeler is 0.6 , and even when the number of labelers increases to 13 , there is still a higher level of noise in the set of integrated labels. Therefore, noise filtering is very necessary for improving crowdsourcing ${ }_{113}$ learning.

Noise filtering is not a new technique in the machine learn-115 ing community, and it can even be stated that noise filtering ${ }_{116}$ is a relatively mature field. Many years ago, researchers al-117 ready noticed the presence of noise in data sets, including fea-118 ture noise and label noise. In this paper, we discuss label noise. ${ }_{119}$ However, to the best of our knowledge, in very few studies has 120 the use of existing noise filters been considered for improving ${ }_{121}$ the quality of integrated labels. Since in existing studies many ${ }_{122}$ methods to handle label noise have been presented, our objec-123 tive was to borrow from the results of these studies to enhance ${ }_{124}$ the crowdsourced data quality, and consequently, improve the ${ }_{125}$ model quality.

The rest of this paper is organized as follows. In Section ${ }_{127}$ 2 , related work on crowdsourcing is introduced. In Section 128

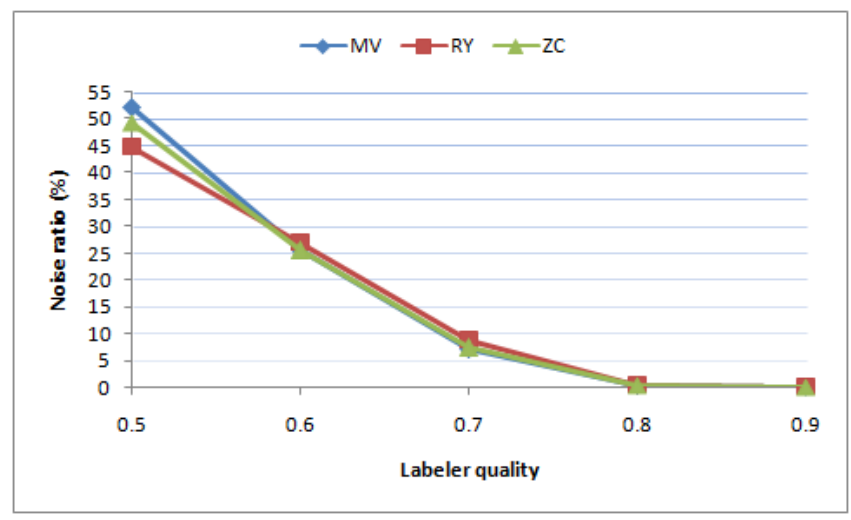

Figure 1: Noise ratio reduces with the increase in labeling qualities and where the number of labelers is 9 .

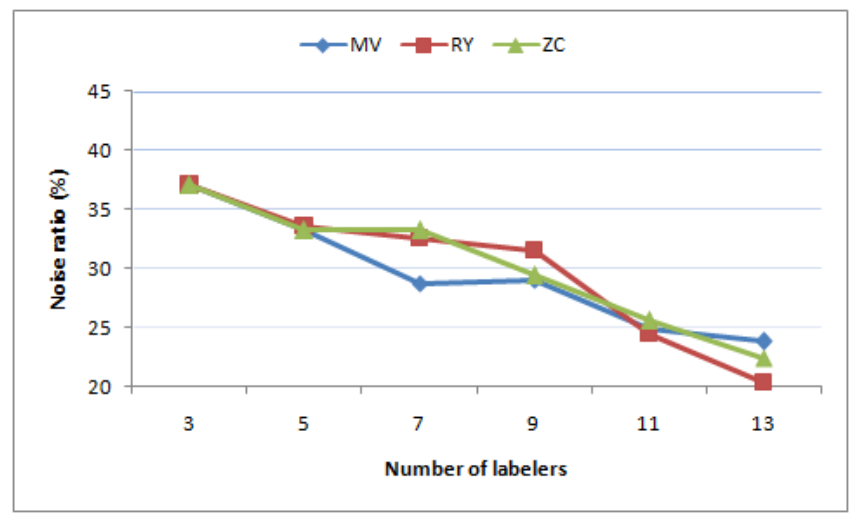

Figure 2: Noise ratio reduces with the increase in the number of labelers and where the labeling quality of each labeler is $p=0.6$.

3, some noise filters are revisited. In Section 4, experimental validation of the performance of these noise filters for deleting noise in integrated labels is presented. Section 5 gives our conclusions and future work.

\section{Related work}

In order to address conventional supervised learning problems in the scenario of crowdsourcing, it is very important to study the induction of an integrated label from multiple noisy labels. Many consensus methods have been designed for label integration, among which MV is the simplest. However, $\mathrm{MV}$ is a little rough. In order to improve the quality of integrated labels, researchers have presented more complicated consensus methods. These can be categorized into two approaches. The first comprises consensus methods based on Expectation Maximization (EM). The common idea of consensus methods based on EM is to use EM to optimize model parameters and estimate labels simultaneously. Representative methods include RY (Raykar et al., 2010), ZC (Demartini et al., 2012), GLAD (Whitehill et al., 2009), and DS (Dawid $\&$ Skene, 1979). The second approach is the weighted majority voting approach, which includes frequency-based major- 
ity voting (MV-Freq), Beta distribution-based majority voting ${ }_{18}$ (MV-Beta) (Sheng, 2011), iterative weighted majority voting ${ }_{187}$ (IWMV), which optimizes the error rate bound and approxi-188 mates the oracle MAP rule ( $\mathrm{Li} \& \mathrm{Yu}, 2014$ ), and max-margin $_{189}$ majority voting $\left(M^{3} V\right)$ (Tian \& Zhu, 2015).

Although these consensus methods perform well in many ${ }_{191}$ real applications, in order to improve crowdsourcing learning, 192 researchers have been attempting to use different techniques to improve the crowdsourced training data quality further, enhance the model performance, and reduce the cost of acquiring labels. Among these techniques, the bridging of crowdsourc-194 ing learning and active learning has attracted many researchers' ${ }_{195}$ attention. Yan et al. (2011) focused on active learning for the ${ }_{196}$ multiple labelers scenario, and their work provided a criterion ${ }_{197}$ for selecting both the most uncertain instance and the labeler/s 198 from whom to query the labels. Zhao et al. (2011) proposed ${ }_{199}$ a modified active learning method that is robust to label noise, 200 because traditional active learning algorithms cannot be suc-201 cessfully applied to crowdsourced training data because of the ${ }_{202}$ problem of label noise. Fang et al. (2012) proposed a new ac-203 tive learning framework, where a self-taught learning system 204 designed to enable labelers to learn complementary knowledge $\mathrm{e}_{205}$ from one another is applied. Zhang et al. (2015) proposed 206 in particular an imbalanced multiple noisy labeling framework $\mathrm{k}_{207}$ based on active learning, where label integration for imbalanced ${ }_{208}$ multiple noisy labeling and instance selection are combined ${ }_{209}$ into a single method.

Another important research direction for improving crowd-211 sourcing learning is to estimate the quality of the crowd work-212 ers. In fact, some consensus methods, such as RY and ZC, can ${ }_{213}$ estimate the quality of crowd workers; they use different param-214 eters to model this quality. In addition, Dawid \& Skene (1979) 215 proposed a method based on an EM algorithm to measure the la-216 beling quality of workers; their method outputs a confusion ma-217 trix listing the error probabilities for each worker. Ipeirotis et al.218 (2010) proposed a method based on the confusion matrix of ${ }_{219}$ each worker to generate a scalar score representing the inherent 220 quality of each worker, where the quality score for each worker 221 can be adjusted for different misclassification costs. Raykar \& 222 Yu (2011) proposed a method to compute a scalar metric based ${ }_{223}$ on the reduction in entropy, which can be used to rank the work-224 ers. By applying these methods to estimate worker quality, we 225 can eliminate low quality workers' labels or weight workers' labels.

Methods that combine crowd-generated multiple noisy la-228 bels and expert-generated labels also constitute an effective ap-22s proach to achieving improvements in crowdsourcing learning ${ }_{230}$ (Kajino et al., 2012; Hu et al., 2014; Wauthier \& Jordan, 2011;231 Liu et al., 2013; Shu et al., 2015). These methods improve 232 crowdsourced data quality by introducing a small number of ${ }_{233}$ expert labels into the training data.

In the studies mentioned above, productive effort was in-235 vested in improving crowdsourcing generalization performance ${ }_{236}$ and promoting the research on crowdsourcing. This paper fo-237 cuses on a new view: using noise filters to improve crowd-238 sourced data quality and model quality. Our work is based on 239 $_{2}$ an obvious fact: a level of noise exists in the set of integrated ${ }_{240}$ labels inferred by consensus methods, but there are few existing works in which noise filtering was used to improve crowdsourcing learning. Our hypothesis is that filtering noise can enhance the data quality and improve the model performance of crowdsourcing. For this purpose, we tested the effectiveness of noise filtering methods when applied to crowdsourced data. In the following section, we review some of these methods.

\section{Noise filters for label noise}

This paper focuses on label noise. In this section, we review some noise filters for handling label noise. As mentioned, the study of noise handling is a relatively mature field, and many techniques for handling label noise have been presented. A comprehensive review of the existing studies on label noise can be found in (Frénay \& Verleysen, 2014). Our goal is to select noise filters for deleting noise in integrated labels. The large number of existing noise filtering algorithms prevents us from presenting them exhaustively. We selected two categories of noise filters and verified that they can enhance the integrated label quality. One category comprises the noise filters based on KNN algorithms and the second the noise filters based on ensemble learning.

Wilson \& Martinez (2000) presented a comprehensive survey of data cleansing techniques based on KNN algorithms and also proposed six new data cleansing techniques, DROP1-6. Note that most of these data cleansing techniques, including DROP1-6, serve more as instance induction algorithms than as serious noise filters. These techniques remove not only noisy instances but also the internal instances, and retain only border points. These instance induction algorithms do not suit our purpose. KNN classifiers may classify query instances well using only a small number of border points, while many classifiers, such as decision tree algorithms, cannot perform well when the number of training instances is too small. Moreover, our purpose was only to remove noise, and therefore, we needed pure noise filters rather than instance induction algorithms. Thus, we selected Edited Nearest Neighbor Rule (ENN) (Wilson, 1972), and its improved version, the All KNN method (Tomek, 1976). The idea of the ENN rule is to use a KNN classifier to remove noisy instances, where an instance $\mathbf{x}$ in a training data set is removed if its label does not agree with the majority of its $k$ nearest neighbors. Algorithm 1 describes the detailed algorithmic procedure of the ENN rule.

As mentioned, All KNN is an improved version of ENN. Unlike ENN, which employs only one classifier to determine whether an instance $\mathbf{x}$ is noise, All KNN uses $k$ nearest neighbor classifiers ( $i \mathrm{NN}$ classifier, $i=1,2, \cdots, k$ ) for this purpose. The procedure of All KNN is as follows. For $i=1$ to $k$, if any of $k$ classifiers misclassifies instance $\mathbf{x}$, then this instance is considered to be noise, and finally all noisy instances are removed from the training data set at once. Only a small simple modification based on the ENN rule needs to be made to the detailed procedure of the All KNN algorithm. We do not show this modification here.

The second category of noise filters we selected comprises those based on ensemble learning. These algorithms in gen- 


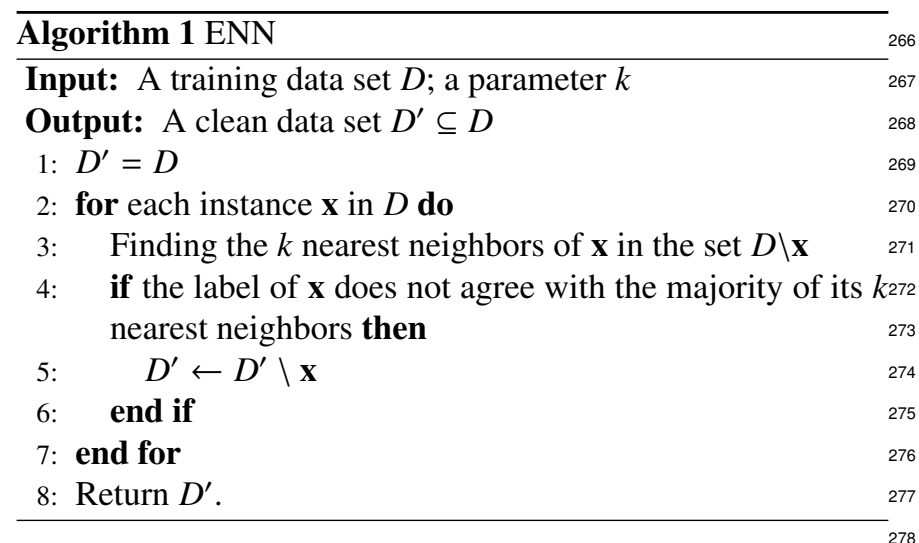

eral partition a training data set into small subsets and build ${ }_{280}$ multiple classifiers on the generated subsets according to dif- ${ }_{281}$ ferent strategies. The multiple classifiers together determine ${ }_{282}$ whether an instance is noise. We chose three filters: the Clas- ${ }_{283}$ sification Filter (Gamberger et al., 1999), Majority Vote Filter ${ }_{284}$ (Brodley, 1999), and Iterative-Partitioning Filter (Khoshgoftaar ${ }_{285}$ \& Rebours, 2007). The idea of the Classification Filter is $\operatorname{as}_{286}$ follows. A training data set is partitioned into $n$ subsets; then, ${ }_{287}$ for each part of $n$ subsets, the part is excluded and a classifier ${ }_{288}$ is built on the remaining $n-1$ subsets. Then, the classifier is ${ }_{289}$ used to predict the label of each instance on the excluded sub- ${ }_{290}$ set. The instances that are misclassified are considered noise ${ }_{291}$ and removed from the training data set. The algorithmic proce- ${ }_{292}$ dure of the Classification Filter is shown in Algorithm 2.

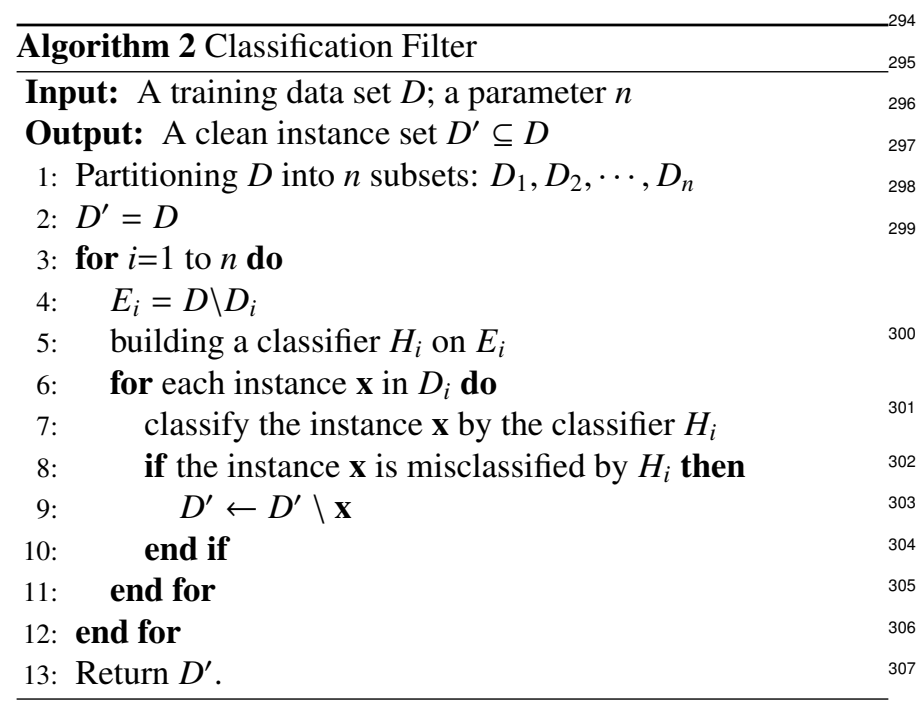

The difference between the Majority Vote Filter and the Clas- ${ }^{308}$ sification Filter is that, for the Majority Vote Filter, after a train- ${ }^{309}$ ing data set is partitioned into $n$ subsets, for each part of $n^{310}$ subsets, a part is excluded and $m(m>1)$ different classifiers ${ }^{311}$ (not a single classifier) are built on the remaining $n-1$ subsets. ${ }^{312}$ Then, when an instance in the excluded subset is misclassified ${ }^{313}$ by more than half of $m$ classifiers, the instance is considered to $\mathrm{o}^{314}$ be noise. The Majority Vote Filter is intended to overcome the ${ }^{315}$ bias of a single classifier. In contrast to the Majority Vote Filter ${ }_{316}$ and the Classification Filter, the Iterative-Partitioning Filter also ${ }_{317}$ partitions a training data set into $n$ subsets, but builds a classi-318 fier on each subset (but does not build a classifier or multiple classifiers on the remaining $n-1$ subsets). Thus, $n$ classifiers are built. Then, the $n$ classifiers are together used to classify all instances in the training data set. An instance is identified as noise if the following two conditions are met: 1. the instance is misclassified by more than half of $n$ classifiers (or is misclassified by all $n$ classifiers); 2 . the instance is misclassified by the classifier that is built on the subset containing the instance. When all noisy instances are removed, a new and clean training data set is obtained. Then, the above steps are repeated for the new data set until the stopping criterion is met. The stopping criterion in (Khoshgoftaar \& Rebours, 2007) is set to be, for three successive iterations, the number of identified noise instances in each iteration that is less than $1 \%$ of the size of the original training data set. The algorithmic procedures of the Majority Vote Filter and Iterative-Partitioning Filter need only simple modifications based on Algorithm 2, and therefore, we do not present them here.

Recently, researchers have also presented some new studies on noise handling. For example, Garcia et al. (2015) proposed a one-vs-one decomposition strategy for noise filtering in multiclass classification problems and Sáez et al. $(2013,2016)$ analyzed the performance and robustness of several multiple classifier systems inducted on noisy data and proposed a noise filtering method that combines multiple filtering strategies based on the fusion of the predictions of multiple filtering strategies used to detect noise. The large number of noise filters presented in existing studies prevents us from listing them one by one in this paper. It is also not the purpose of this paper to list as many existing noise filters as possible. Our objective was only to select some filters and apply them to crowdsourcing learning and provide researchers with a new idea for improving crowdsourcing learning. In the next section, we describe the experimental verification of the effectiveness of these noise filters.

\section{Experiments and results}

\subsection{Experimental setting and benchmark data}

The purpose of the experiments described in this section was to test the performance of the five noise filters described in Section 3 in terms of the NR of data sets and the classification accuracy of target classifiers. The target classifier was set to be C4.5 (Quinlan, 1993). The settings of the five noise filters and their abbreviations are introduced as follows.

- ENN, All KNN: only one parameter $k$ has to be set; in our experiments, $k=3$ (Wilson, 1972; Tomek, 1976).

- CF: Classification Filter (Gamberger et al., 1999). To apply the filter, the parameter $n$ ( $n$ is the number of subsets into which a training data set is partitioned) and the classifier that serves as the filter need to be set. In our experiments, $n=10$, and the classifier serving as the filter was C4.5.

- MVF: Majority Vote Filter (Brodley, 1999). To apply the filter, two parameters, $n$ and $m$ ( $n$ is the number of subsets into which the training data set is partitioned, and $m$ is 
Table 1: Descriptions of 14 UCI data sets used in the experiments

\begin{tabular}{lcccc}
\hline Dataset & Attributes & Examples & Positives & Negatives \\
\hline biodeg & 41 & 1055 & 356 & 699 \\
breast-cancer & 9 & 268 & 85 & 201 \\
breast-w & 9 & 699 & 241 & 458 \\
credit-a & 15 & 690 & 383 & 307 \\
credit-g & 20 & 1000 & 300 & 700 \\
heart-statlog & 13 & 270 & 120 & 150 \\
hepatitis & 19 & 155 & 123 & 32 \\
horse-colic & 22 & 368 & 232 & 136 \\
ionosphere & 34 & 351 & 225 & 126 \\
kr-vs-kp & 36 & 3196 & 1527 & 1669 \\
mushroom & 22 & 8123 & 3916 & 4208 \\
sick & 29 & 3772 & 231 & 3541 \\
sonar & 60 & 208 & 111 & 97 \\
spambase & 57 & 4601 & 1813 & 2788 \\
\hline
\end{tabular}

the number of classifiers that serve as the filters), and the classifiers that serve as the filters need to be set. In our experiments, $n=4, m=3$, and the classifiers serving as the filters were 1-NN, C4.5, and NB (naive Bayes classifier) (Wu et al., 2008).

- IPF: Iterative-Partitioning Filter (Khoshgoftaar \& Rebours, 2007). To apply the filter, the parameter $n$ ( $n$ is the number of subsets into which the training data set is partitioned), the classifier serving as the filter, and the voting strategy that is used to identify noise need to be set. In our experiments, $n=5$, the classifier serving as the filter was $\mathrm{C} 4.5$, and an instance was identified as noise if more than half of the classifiers misclassified it.

Note that all settings for parameters $k, n$, and $m$ were the same as those reported in the papers in which these algorithms were originally presented. We used the existing implementations of KNN, C4.5, and NB in WEKA (Witten et al., 2011) and implemented all the other algorithms. We ran our experiments ${ }^{357}$ on 14 binary classification benchmark data sets published by 358 UCI (Frank \& Asuncion, 2010), which represent a wide range359 of domains and data characteristics. A detailed description of 360 these UCI data sets is shown in Table 1.

In order to simulate a crowdsourcing process to obtain mul-362 tiple noisy labels of each instance, the original true labels of ${ }^{363}$ all the instances were hidden, and nine simulated labelers were ${ }^{364}$ employed to label each instance. For each labeler, the original ${ }^{365}$ true label was assigned to each instance with probability $p_{j}$ and ${ }^{366}$ the opposite value was assigned with probability $1-p_{j}$. T0 ${ }^{367}$ determine the robustness of the experimental results to differ-368 ent labeling qualities, two different labeling quality setups were ${ }^{369}$ considered:

1. In the first series of experiments, all labelers' labeling ${ }_{372}$ qualities were fixed at 0.6. Namely, $p_{j}=0.6(j=$ $1,2, \ldots, 9)$.

2. In the second series of experiments, the labeling qual- ${ }_{374}$ ity of each labeler was generated randomly from a uni- ${ }_{375}$ form distribution on the interval $[0.55,0.75]$. Namely, ${ }_{376}$ $p_{j} \in[0.55,0.75](j=1,2, \ldots, 9)$.
Table 2: Noise ratio (\%) comparisons in the first series of experiments, where all labelers' labeling qualities were fixed at 0.6

\begin{tabular}{lcccccc}
\hline Dataset & No & ENN & All KNN & CF & MVF & IPF \\
\hline biodeg & 25.88 & 14.94 & 15.24 & 12.7 & 11.63 & 7.33 \\
breast-cancer & 31.82 & 22.78 & 22.17 & 19.08 & 18.82 & 15.19 \\
breast-w & 27.04 & 8.66 & 9 & 4.07 & 2.36 & 2.02 \\
credit-a & 25.94 & 16.81 & 14.09 & 8.53 & 8.16 & 5.76 \\
credit-g & 25.2 & 19.51 & 18.77 & 15.32 & 13.55 & 13.05 \\
heart-statlog & 24.81 & 15.12 & 14.39 & 14.36 & 10.31 & 11.67 \\
hepatitis & 27.1 & 24.37 & 19.58 & 15.21 & 9.98 & 9.36 \\
horse-colic & 28.26 & 21.93 & 24.32 & 7.23 & 11.79 & 6.6 \\
ionosphere & 27.92 & 17.29 & 18.37 & 9.89 & 9.33 & 10.03 \\
kr-vs-kp & 27.47 & 12.69 & 12.36 & 2.59 & 3.66 & 2.47 \\
mushroom & 27.15 & 5.07 & 6.61 & 0.08 & 0.73 & 0.32 \\
sick & 27.89 & 13.45 & 10.12 & 2.87 & 9.78 & 1.1 \\
sonar & 27.41 & 17.46 & 17.22 & 21.12 & 17.01 & 15.5 \\
spambase & 28.71 & 16.7 & 15.22 & 5.62 & 7.41 & 4.29 \\
\hline Average & 27.33 & 16.2 & 15.53 & 9.91 & 9.61 & 7.48 \\
\hline
\end{tabular}

Table 3: Classification accuracy (\%) comparisons in the first series of experiments, where all labelers' labeling qualities were fixed at 0.6

\begin{tabular}{lcccccc}
\hline Dataset & No & ENN & All KNN & CF & MVF & IPF \\
\hline Biodeg & 69.29 & 73.35 & 71.23 & 76.2 & 76.66 & 74.91 \\
Breast-cancer & 62.67 & 70.44 & 69.01 & 70.99 & 68.13 & 70.08 \\
Breast-w & 89.45 & 93.64 & 93.98 & 90.94 & 93.64 & 93.66 \\
Credit-a & 76.96 & 81.59 & 81.01 & 78.55 & 79.28 & 85.65 \\
Credit-g & 65 & 66.8 & 65.9 & 68.2 & 68.8 & 70 \\
Heart-statlog & 70 & 72.22 & 70.74 & 71.48 & 71.85 & 73.7 \\
Hepatitis & 75.5 & 81.17 & 77 & 75.33 & 80.67 & 80 \\
Horse-colic & 82.8 & 79.57 & 74.19 & 84.75 & 84.75 & 83.08 \\
Ionosphere & 79.14 & 73.21 & 73.71 & 83.71 & 82.58 & 85.44 \\
Kr-vs-kp & 86.66 & 83.54 & 85.72 & 89.47 & 88.57 & 91.55 \\
Mushroom & 97.9 & 99.13 & 98.43 & 99.08 & 98.91 & 98.38 \\
Sick & 92.95 & 95.04 & 95.92 & 96.18 & 95.89 & 97.85 \\
Sonar & 50.86 & 55.43 & 55.14 & 52.64 & 61.36 & 59.57 \\
Spambase & 82.53 & 77.27 & 76.88 & 85.79 & 83.79 & 86.22 \\
\hline Average & 77.23 & 78.74 & 77.78 & 80.24 & 81.06 & 82.15 \\
\hline
\end{tabular}

After obtaining multiple noisy labels of each instance, the consensus method MV was applied to infer integrated labels. The example presented in Section 1 shows that the various consensus methods always result in a level of noise in the set of integrated labels, and therefore, in our experiments we applied only the simplest consensus method, MV, to infer the integrated labels of instances. After acquiring the integrated labels of all the instances, the five noise filters were applied to all 14 data sets, and then the NR of the integrated labels and the classification accuracy of target classifiers based on different noise filters applied to each data set were obtained via 10-fold crossvalidation. The various algorithms were run on the same training sets and evaluated on the same test sets. Note that the test sets were not involved in the calculation of the NR. In particular, the cross-validation folds were the same for all algorithms on each data set.

\subsection{Experimental results and analysis}

First, we present the experimental results of the first series of experiments, where all labelers' labeling qualities were the same. Table 2 shows the NR in the original integrated labels and the NR after the noise filters were applied to each data set. 
"No" denotes the results when noise filters were not applied. The experimental results in Table 2 show that all the noise filters significantly reduce the NR of each data set. Can the reduction in the NR improve the classification performance of target classifiers? Table 3 shows the detailed classification accuracy of the target classifiers based on different noise filters applied to each data set. "No" denotes the results when no noise filter was applied.

These experimental results prove powerfully the effectiveness of noise filters for improving data and model quality. We can draw the following specific conclusions.

1. A low individual labeling quality (in our experiments, $p=0.6$ for each labeler) and relatively high original NRs in the 14 data sets (all reached above $25 \%$ except for one) demonstrate that a certain level of label noise exists in the set of integrated labels inferred by consensus methods. Noise filters are very necessary for crowdsourcing learning.

2. For all 14 data sets, the five noise filters all significantly reduce the NR of integrated labels. The average NR for the 14 data sets before noise filtering is $27.33 \%$. The average NRs after applying ENN and All KNN are $16.2 \%$ and $15.53 \%$, respectively, and the average NRs after applying CF, MVF, and IPF are $9.91 \%, 9.61 \%$ and $7.48 \%$, respectively. In general, CF, MVF, and IPF can delete more noisy instances than can ENN and All KNN. For 8 of the 14 data sets, CF, MVF, and IPF can all reduce the NR to a level under $10 \%$.

3. The five noise filters all significantly reduce the NR of integrated labels for almost all data sets, and consequently, the classification performance of the target classifiers for almost all the data sets shows a noteworthy improvement as compared with the classifiers induced on data sets without the application of noise filters. The average classification accuracy for the 14 data sets before noise filtering is $77.23 \%$, and the average classification accuracy when ENN and All KNN are applied is $78.74 \%$ and $77.78 \%$, respectively. The average classification accuracy after ap- ${ }^{435}$ plying CF, MVF, and IPF is $80.24 \%, 81.06 \%$, and $82.15 \%$ respectively.

4. In terms of NR and classification accuracy, the three noise ${ }^{438}$ filters based on ensemble learning generally show a bet- ${ }^{439}$ ter performance than the two noise filters based on $\mathrm{KNN}^{440}$ algorithms.

5. The data set mushroom is distinctive. Surprisingly, when ${ }^{442}$ the NR of the integrated labels of the data set reaches $27.15 \%$, the classification performance of C4.5 induced on ${ }^{44}$ the data set still achieves $97.9 \%$ accuracy. According to ${ }^{445}$ our observation, this data set is very large (8123 instances) ${ }^{446}$ and is balanced. The introduction of random and uniform ${ }^{447}$ label noise into multiple noisy labels does not change the ${ }^{448}$ class distribution of data and also does not strongly influ- ${ }^{449}$ ence the process of building C4.5.

Next, we present the experimental results of the second series 452 of experiments, where the labeling quality of each labeler was ${ }_{453}$ generated randomly from an uniform distribution on the interval ${ }_{454}$
Table 4: Noise ratio (\%) comparisons for the second series of experiments, where each labeler's labeling quality was generated randomly from an uniform distribution on the interval $[0.55,0.75]$

\begin{tabular}{lcccccc}
\hline Dataset & No & ENN & All KNN & CF & MVF & IPF \\
\hline biodeg & 14.31 & 5.48 & 5.72 & 4.12 & 3.51 & 2.54 \\
breast-cancer & 14.69 & 6.56 & 7.31 & 5.48 & 5.35 & 6.1 \\
breast-w & 25.89 & 8 & 8.97 & 3.91 & 2.45 & 1.52 \\
credit-a & 16.81 & 8.52 & 8.13 & 4.54 & 4.23 & 4.53 \\
credit-g & 21.4 & 14.57 & 13.86 & 12.17 & 9.81 & 10.1 \\
heart-statlog & 16.67 & 10.85 & 11.04 & 7.84 & 6.32 & 5.82 \\
hepatitis & 17.42 & 8.79 & 9.56 & 6.77 & 5.25 & 5.88 \\
horse-colic & 25.27 & 12.59 & 13.75 & 9.95 & 9.39 & 12.31 \\
ionosphere & 15.95 & 6.83 & 7.65 & 3.87 & 3.89 & 4.25 \\
kr-vs-kp & 16.4 & 4.31 & 4.3 & 0.36 & 0.95 & 0.54 \\
mushroom & 13.18 & 0.41 & 0.8 & 0.01 & 0.13 & 0.13 \\
sick & 18.32 & 5.34 & 3.62 & 0.53 & 0.8 & 0.61 \\
sonar & 19.23 & 8.44 & 7.94 & 11.54 & 5.16 & 5.59 \\
spambase & 14.45 & 4.47 & 4.3 & 2.01 & 2.31 & 1.59 \\
\hline Average & 17.86 & 7.51 & 7.64 & 5.22 & 4.25 & 4.39 \\
\hline
\end{tabular}

Table 5: Classification accuracy (\%) comparisons for the second series of experiments, where (each labeler's labeling quality was generated randomly from an uniform distribution on the interval $[0.55,0.75])$

\begin{tabular}{lcccccc}
\hline Dataset & No & ENN & All KNN & CF & MVF & IPF \\
\hline biodeg & 72.7 & 77.6 & 77.43 & 75.36 & 78.48 & 76.42 \\
breast-cancer & 69.5 & 70.63 & 72.23 & 70.63 & 73.59 & 70.08 \\
breast-w & 87.67 & 92.69 & 89.94 & 92.98 & 93.08 & 94.24 \\
credit-a & 83.62 & 83.48 & 84.93 & 83.33 & 85.8 & 85.51 \\
credit-g & 65.6 & 71.4 & 71.9 & 69.9 & 71.8 & 69.8 \\
heart-statlog & 71.85 & 74.81 & 73.33 & 78.15 & 80 & 76.3 \\
hepatitis & 72.17 & 76.33 & 76.83 & 81.17 & 82.67 & 80 \\
horse-colic & 80.96 & 83.86 & 83.91 & 81.97 & 83.91 & 76.97 \\
ionosphere & 78.04 & 82.87 & 82.9 & 83.18 & 85.73 & 86.87 \\
kr-vs-kp & 93.67 & 92.43 & 86.43 & 95.85 & 95.49 & 92.17 \\
mushroom & 99.64 & 99.9 & 99.93 & 99.64 & 99.86 & 98.65 \\
sick & 97.19 & 97.27 & 97.19 & 98.09 & 97.91 & 97.75 \\
sonar & 53.21 & 58.79 & 45.79 & 54.07 & 56.07 & 62.57 \\
spambase & 84.07 & 85.31 & 84.77 & 87.48 & 87.22 & 88.79 \\
\hline Average & 79.28 & 81.96 & 80.54 & 82.27 & 83.69 & 82.58 \\
\hline
\end{tabular}

$[0.55,0.75]$. Table 4 and Table 5 show the detailed comparison results in terms of the NR and classification accuracy, respectively. From these comparison results, we can draw almost the same conclusions. To avoid repetition, we do not explain these results in detail here.

In addition, we used the KEEL Data-Mining Software Tool (Alcalá-Fdez et al., 2011) to conduct a Friedman test with the corresponding post-hoc tests (Demsar, 2006; García \& Herrera, 2008), such as the Bergmann test, for the comparison of more classifiers over multiple data sets. Tables 6, 8, 10, and 12 show the average rankings of the algorithms obtained by applying the Friedman test (Demsar, 2006; García \& Herrera, 2008), respectively. For the 6 algorithms and 14 data sets, $F_{F}$ is distributed according to the $F$ distribution with $6-1=5$ and $(6-1) \times(14-1)=65$ degrees of freedom. $F_{F}$ calculated from the mean ranks is $90.697674,8.756526,62.004205$, and 7.591088 , respectively, all of which values are greater than the critical values of $F(5,65)$ for $a=0.1$. Therefore, we rejected the null hypothesis and proceeded to the Bergmann test to determine accurately the significant difference between each pair of algorithms. Tables 7, 9, 11, and 13 respectively show the 
Table 6: Average rankings of the algorithms obtained by applying the Friedman test: Noise ratio (\%) comparisons for the first series of experiments

\begin{tabular}{ll}
\hline Algorithm & Ranking \\
\hline No & 6 \\
ENN & 4.5714 \\
All KNN & 4.2857 \\
CF & 2.6429 \\
MVF & 2.2143 \\
IPF & 1.2857 \\
\hline
\end{tabular}

$z$-values and the $p$-values obtained, where the detailed results using the Bergmann test indicate which pairs of algorithms are significantly different. These test results show that:

1. According to the Friedman test results in Tables 6, 8, 10, and 12, the average rankings of the five noise filters ENN, All KNN, CF, MVF, and IPF are always higher than the model in which noise filtering is not applied. Furthermore, the average rankings of CF, MVF, and IPF are higher than those of ENN and All KNN.

2. According to the post-hoc Bergmann test results shown in Tables 7, 9, 11, and 13, in terms of both NR and classification accuracy, CF, MVF, and IPF all significantly outperform the model in which noise filters are not applied. For ENN and All KNN, sometimes the differences between ENN, All KNN, and the model in which noise filtering is not applied are not very prominent. For example, Table 11 shows that, in terms of NR, there are no significant differences between ENN, All KNN, and the model in which noise filters are not applied. However, Table 13 shows that in terms of classification accuracy, ENN and All KNN still significantly outperform the model in which noise filters are not applied. In general, ENN and All KNN still outperform the model in which noise filtering is no applied.

\subsection{Experiments on real-world data}

Finally, to further test the performance of noise filters in terms of improving crowdsourcing learning, we ran experiments on three real-world data sets, which were collected from Amazon Mechanical Turk. We knew in advance the true labels of all the instances. The detailed data characteristics of these three real-world data sets are shown in Table 14.

Figures 3 and 4 respectively show the comparison results for NR and classification accuracy based on different noise filters. According to these experimental results, we can reach conclusions similar to those drawn from the results of experiments on the benchmark UCI data sets.

\section{Conclusions and future work}

The main purpose of the study presented in this paper was to investigate the performance of noise filters for crowdsourcing learning. In existing papers, many methods to handle label noise have been presented, and therefore, in this study we attempted to use previous research achievements to improve crowdsourced data and model quality. We revisited several
Table 7: P-values for $\alpha=0.1$ : Noise ratio (\%) comparisons for the first series of experiments

\begin{tabular}{llll}
\hline$i$ & algorithms & $z=\left(R_{0}-R_{i}\right) / S E$ & $p$ \\
\hline 15 & No vs. IPF & 6.667007 & 0 \\
14 & No vs. MVF & 5.353808 & 0 \\
13 & No vs. CF & 4.747717 & 0.000002 \\
12 & ENN vs. IPF & 4.646702 & 0.000003 \\
11 & All KNN vs. IPF & 4.242641 & 0.000022 \\
10 & ENN vs. MVF & 3.333503 & 0.000858 \\
9 & All KNN vs. MVF & 2.929442 & 0.003396 \\
8 & ENN vs. CF & 2.727412 & 0.006383 \\
7 & No vs. All KNN & 2.424366 & 0.015335 \\
6 & All KNN vs. CF & 2.323351 & 0.02016 \\
\hline 5 & No vs. ENN & 2.020305 & 0.043352 \\
4 & CF vs. IPF & 1.91929 & 0.054948 \\
3 & MVF vs. IPF & 1.313198 & 0.189116 \\
2 & CF vs. MVF & 0.606092 & 0.544454 \\
1 & ENN vs. All KNN & 0.404061 & 0.686168 \\
\hline
\end{tabular}

Bergmann's procedure rejects these hypotheses:

- No vs. All KNN

- No vs. CF

- No vs. MVF

- No vs. IPF

- ENN vs. CF

- ENN vs. MVF

- ENN vs. IPF

- All KNN vs. CF

- All KNN vs. MVF

- All KNN vs. IPF

Table 8: Average rankings of the algorithms obtained by applying the Friedman test: Classification accuracy $(\%)$ comparisons for the first series of experiments

\begin{tabular}{ll}
\hline Algorithm & Ranking \\
\hline No & 5.3571 \\
ENN & 3.5357 \\
All KNN & 4.2857 \\
CF & 3.0357 \\
MVF & 2.7857 \\
IPF & 2 \\
\hline
\end{tabular}

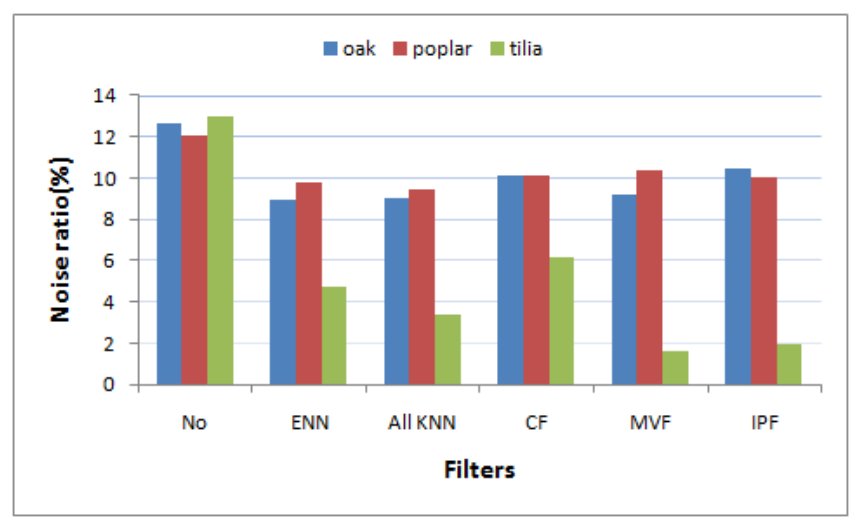

Figure 3: Noise ratio comparisons for three real-world data sets 
Table 9: P-values for $\alpha=0.1$ : Classification accuracy (\%) comparisons in the first series of experiments.

\begin{tabular}{llll}
\hline$i$ & algorithms & $z=\left(R_{0}-R_{i}\right) / S E$ & $p$ \\
\hline 15 & No vs. IPF & 4.747717 & 0.000002 \\
14 & No vs. MVF & 3.636549 & 0.000276 \\
13 & No vs. CF & 3.282996 & 0.001027 \\
12 & All KNN vs. IPF & 3.232488 & 0.001227 \\
11 & No vs. ENN & 2.575889 & 0.009998 \\
\hline 10 & ENN vs. IPF & 2.171828 & 0.029869 \\
9 & All KNN vs. MVF & 2.12132 & 0.033895 \\
8 & All KNN vs. CF & 1.767767 & 0.0771 \\
7 & No vs. All KNN & 1.515229 & 0.129714 \\
6 & CF vs. IPF & 1.464721 & 0.142997 \\
5 & MVF vs. IPF & 1.111168 & 0.266496 \\
4 & ENN vs. MVF & 1.06066 & 0.288844 \\
3 & ENN vs. All KNN & 1.06066 & 0.288844 \\
2 & ENN vs. CF & 0.707107 & 0.4795 \\
1 & CF vs. MVF & 0.353553 & 0.723674 \\
\hline
\end{tabular}

The results of Bergmann's procedure reject these hypotheses:

- No vs. ENN

- No vs. CF

- No vs. MVF

- No vs. IPF

- All KNN vs. IPF

Table 10: Average rankings of the algorithms obtained by applying the Friedman test: Noise ratio (\%) comparisons for the second series of experiments.

\begin{tabular}{ll}
\hline Algorithm & Ranking \\
\hline No & 6 \\
ENN & 4.3571 \\
All KNN & 4.5 \\
CF & 2.3571 \\
MVF & 1.8214 \\
IPF & 1.9643 \\
\hline
\end{tabular}

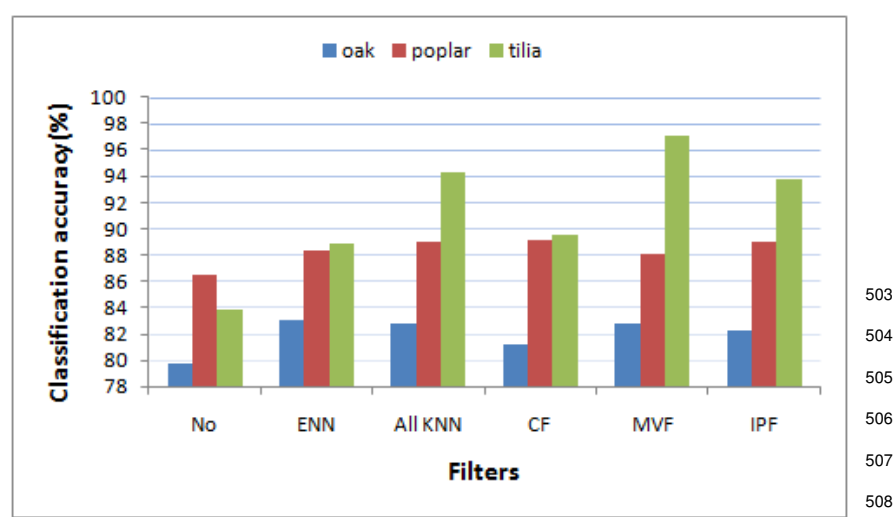

Figure 4: Classification accuracy comparisons for three real-world data sets.

noise filters and applied them to crowdsourced data. The ex-510 perimental results based on benchmark and real-world data sets $s_{511}$
Table 11: P-values for $\alpha=0.1$ : Noise ratio (\%) comparisons for the second series of experiments

\begin{tabular}{llll}
\hline$i$ & algorithms & $z=\left(R_{0}-R_{i}\right) / S E$ & $p$ \\
\hline 15 & No vs. MVF & 5.909392 & 0 \\
14 & No vs. IPF & 5.707362 & 0 \\
13 & No vs. CF & 5.151778 & 0 \\
12 & All KNN vs. MVF & 3.788072 & 0.000152 \\
11 & All KNN vs. IPF & 3.586042 & 0.000336 \\
10 & ENN vs. MVF & 3.586042 & 0.000336 \\
9 & ENN vs. IPF & 3.384011 & 0.000714 \\
8 & All KNN vs. CF & 3.030458 & 0.002442 \\
7 & ENN vs. CF & 2.828427 & 0.004678 \\
\hline 6 & No vs. ENN & 2.323351 & 0.02016 \\
5 & No vs. All KNN & 2.12132 & 0.033895 \\
4 & CF vs. MVF & 0.757614 & 0.448682 \\
3 & CF vs. IPF & 0.555584 & 0.578495 \\
2 & ENN vs. All KNN & 0.202031 & 0.839893 \\
1 & MVF vs. IPF & 0.202031 & 0.839893 \\
\hline
\end{tabular}

The results of Bergmann's procedure reject these hypotheses:

- No vs. CF

- No vs. MVF

- No vs. IPF

- ENN vs. CF

- ENN vs. MVF

- ENN vs. IPF

- All KNN vs. CF

- All KNN vs. MVF

- All KNN vs. IPF

Table 12: Average rankings of the algorithms obtained by applying the Friedman test: Classification accuracy (\%) comparisons for the second series of experiments

\begin{tabular}{ll}
\hline Algorithm & Ranking \\
\hline No & 5.3571 \\
ENN & 3.6071 \\
All KNN & 3.7143 \\
CF & 3.2143 \\
MVF & 1.8214 \\
IPF & 3.2857 \\
\hline
\end{tabular}

duced from multiple noise labels and are also affected by certain factors, such as the labeling quality of labeler and the instance difficulty. We believe that considering more factors that affect noise filtering systems in the design of fine noise filters can improve their performance further. This is one of our main future work directions.

\section{Acknowledgements}

The work was partially supported by the National Natural Science Foundation of China (61203287), the U.S. National Science Foundation under the grant IIS-1115417, the Program for New Century Excellent Talents in University (NCET-120953), and the Chenguang Program of Science and Technology of Wuhan (2015070404010202). 
Table 13: P-values for $\alpha=0.1$ : Classification accuracy (\%) comparisons for ${ }_{544}^{543}$ the second series of experiments

\begin{tabular}{llll}
\hline$i$ & algorithms & $z=\left(R_{0}-R_{i}\right) / S E$ & $p$ \\
\hline 15 & No vs. MVF & 5.000255 & 0.000001 \\
14 & No vs. CF & 3.030458 & 0.002442 \\
13 & No vs. IPF & 2.929442 & 0.003396 \\
12 & All KNN vs. MVF & 2.676904 & 0.007431 \\
11 & ENN vs. MVF & 2.525381 & 0.011557 \\
10 & No vs. ENN & 2.474874 & 0.013328 \\
9 & No vs. All KNN & 2.323351 & 0.02016 \\
\hline 8 & MVF vs. IPF & 2.070813 & 0.038376 \\
7 & CF vs. MVF & 1.969797 & 0.048862 \\
6 & All KNN vs. CF & 0.707107 & 0.4795 \\
5 & All KNN vs. IPF & 0.606092 & 0.544454 \\
4 & ENN vs. CF & 0.555584 & 0.578495 \\
3 & ENN vs. IPF & 0.454569 & 0.64942 \\
2 & ENN vs. All KNN & 0.151523 & 0.879563 \\
1 & CF vs. IPF & 0.101015 & 0.919538 \\
\hline
\end{tabular}

The results of Bergmann's procedure reject these hypotheses:

- No vs. ENN

- No vs. All KNN

- No vs. CF

- No vs. MVF

- No vs. IPF

- ENN vs. MVF

- All KNN vs. MVF

Table 14: Descriptions of three real-world data sets used in the experiments

\begin{tabular}{lccccc}
\hline Dataset & Attributes & Examples & Labelers & Positives & Negatives \\
\hline Oak & 64 & 384 & 10 & 96 & 288 \\
Poplar & 64 & 384 & 10 & 48 & 336 \\
Tilia & 64 & 141 & 10 & 45 & 96 \\
\hline
\end{tabular}

\section{References}

Alcalá-Fdez, J., Fernandez, A., Luengo, J., Derrac, J., García, S., Sánchez, L., , 589 \& Herrera, F. (2011). KEEL data-mining software tool: data set repository, integration of algorithms and experimental analysis framework. Journal of Multiple-Valued Logic and Soft Computing, 17(2-3), 255-287.

Brodley, M. A., C. E.and Friedl (1999). Identifying mislabeled training data. ${ }_{593}$ Journal of Artificial Intelligence Research, (pp. 131-167).

Dawid, A. P., \& Skene, A. M. (1979). Maximum likelihood estimation of ob- ${ }_{595}^{594}$ server error-rates using the em algorithm. Applied Statistics, 28(1), 20-28. ${ }_{596}$

Demartini, G., Difallah, D. E., \& Cudré-Mauroux, P. (2012). Zencrowd: lever- ${ }_{597}$ aging probabilistic reasoning and crowdsourcing techniques for large-scale ${ }_{598}$ entity linking. In Proceedings of the 21st International Conference on World 599 Wide Web (pp. 469-478). ACM.

Demsar, J. (2006). Statistical comparisons of classifiers over multiple data sets. ${ }_{601}{ }_{601}$ Journal of Machine Learning Research, 7, 1-30.

Fang, M., Zhu, X., Li, B., Ding, W., \& Wu, X. (2012). Self-taught active learning from crowds. In 2012 IEEE 12th International Conference on Data ${ }_{604}$ Mining (ICDM), (pp. 858-863). IEEE.

Frank, A., \& Asuncion, A. (2010). UCI machine learning repository. Depart- ${ }_{606}{ }_{606}$ ment of Information and Computer Science, University of California, Irvine. ${ }_{607}$

Frénay, B., \& Verleysen, M. (2014). Classification in the presence of label ${ }_{608}$ noise: a survey. IEEE Transactions on Neural Networks and Learning Sys- ${ }_{609}$ tems, 25(5), 845-869.

Gamberger, D., Lavrac, N., \& Groselj, C. (1999). Experiments with noise ${ }_{611}^{610}$ filtering in a medical domain. In ICML (pp. 143-151). Garcia, L., Sáez, J., Luengo, J., Lorena, A., de Carvalho, A., \& Herrera, F. ${ }_{613}^{612}$ (2015). Using the one-vs-one decomposition to improve the performance of class noise filters via an aggregation strategy in multi-class classification problems. Knowledge-Based Systems, 90, 153-164.

García, S., \& Herrera, F. (2008). An extension on statistical comparisons of classifiers over multiple data sets for all pairwise comparisons. Journal of Machine Learning Research, 9, 2677-2694.

Guo, X., Wang, H., \& Song, Y. e. a. (2014). Brief survey of crowdsourcing for data mining. Expert Systems with Applications, 41(17), 7987-7994.

Hu, Q., He, Q., \& Huang, H. e. a. (2014). Learning from crowds under experts' supervision. In Advances in Knowledge Discovery and Data Mining (pp. 200-211). Springer International Publishing.

Ipeirotis, P. G., Provost, F., \& Wang, J. (2010). Quality management on amazon mechanical turk. In Proceedings of the ACM SIGKDD Workshop on Human Computation (pp. 64-67). ACM.

Kajino, H., Tsuboi, Y., Sato, I., \& Kashima, H. (2012). Learning from crowds and experts. In Workshops at the Twenty-Sixth AAAI Conference on Artificial Intelligence.

Khoshgoftaar, T. M., \& Rebours, P. (2007). Improving software quality prediction by noise filtering techniques. Journal of Computer Science and Technology, 22(3), 387-396.

Li, H., \& Yu, B. (2014). Error rate bounds and iterative weighted majority voting for crowdsourcing. In http://arxiv.org/abs/1411.4086.

Liu, Q., Steyvers, M., Fisher, J. W., \& Ihler, A. (2013). On reliable crowdsourcing and the use of ground truth information. Technical Report Workshop on Crowdsourcing at Scale, HCOMP.

Quinlan, J. R. (1993). C4. 5: programs for machine learning. San Mateo: Morgan Kaufmann.

Raykar, V. C., \& Yu, S. (2011). Ranking annotators for crowdsourced labeling tasks. In Advances in Neural Information Processing Systems (pp. 18091817).

Raykar, V. C., Yu, S., Zhao, L. H., Valadez, G. H., Florin, C., Bogoni, L., \& Moy, L. (2010). Learning from crowds. The Journal of Machine Learning Research, 11, 1297-1322.

Sáez, J. A., Galar, M., Luengo, J., \& Herrera, F. (2013). Tackling the problem of classification with noisy data using multiple classifier systems: analysis of the performance and robustness. Information Sciences, 247, 1-20.

Sáez, J. A., Galar, M., Luengo, J., \& Herrera, F. (2016). Inffc: An iterative class noise filter based on the fusion of classifiers with noise sensitivity control. Information Fusion, 27, 19-32.

Sheng, V. S. (2011). Simple multiple noisy label utilization strategies. In 2011 IEEE 11th International Conference on Data Mining (p. 635C644).

Sheng, V. S., Provost, F., \& Ipeirotis, P. G. (2008). Get another label? improving data quality and data mining using multiple, noisy labelers. In Proceedings of the 14th ACM SIGKDD International Conference on Knowledge Discovery and Data Mining (pp. 614-622). ACM.

Sheshadri, A., \& Lease, M. (2013). Square: A benchmark for research on computing crowd consensus. In Proceedings of First AAAI Conference on Human Computation and Crowdsourcing.

Shu, Z., Sheng, V. S., Wang, D., \& Zhang, J. (2015). Integrating active learning with supervision for crowdsourcing generalization. In Proceedings of the 14th IEEE International Conference on Machine Learning and Applications.

Tian, T., \& Zhu, J. (2015). Max-margin majority voting for learning from crowds. In Advances in Neural Information Processing Systems (p. $1612 \mathrm{C} 1620)$.

Tomek, I. (1976). An experiment with the edited nearest-neighbor rule. IEEE Transactions on Systems, Man, and Cybernetics, (6), 448-452.

Wauthier, F. L., \& Jordan, M. I. (2011). Bayesian bias mitigation for crowdsourcing. In Advances in Neural Information Processing Systems (pp. 1800 1808).

Whitehill, J., Wu, T. F., Bergsma, J., Movellan, J. R., \& Ruvolo, P. L. (2009). Whose vote should count more: Optimal integration of labels from labelers of unknown expertiseg. In Advances in Neural Information Processing Systems (p. 2035C2043).

Wilson, D. L. (1972). Asymptotic properties of nearest neighbor rules using edited data. IEEE Transactions on Systems, Man and Cybernetics, (3), 408421.

Wilson, D. R., \& Martinez, T. R. (2000). Reduction techniques for instancebased learning algorithms. Machine learning, 38(3), 257-286.

Witten, I. H., Frank, E., \& Hall, M. A. (2011). Data mining: practical machine learning tools and techniques. (Third edition ed.). Morgan Kaufmann.

Wu, X., Kumar, V., R., Q. J., Ghosh, J., Yang, Q., Motoda, H., J., M. G., Ng, 
A., Liu, B., Yu, P. S., Zhou, Z., Steinbach, M., Hand, D. J., \& Steinberg, D. (2008). Top 10 algorithms in data mining. Knowledge and Information System, 14(1), 1-37.

Yan, Y., Rosales, R., Fung, G., , \& Dy, J. G. (2011). Active learning from crowds. In Proceedings of the 28th International Conference on Machine Learning (pp. 1161-1168)

Zhang, J., Wu, X., \& Shengs, V. S. (2015). Active learning with imbalanced multiple noisy labeling. IEEE Transactions on Cybernetics, 45(5), 10811093.

Zhao, L., Sukthankar, G., \& Sukthankar, R. (2011). Incremental relabeling for active learning with noisy crowdsourced annotations. In Privacy, Security, Risk and Trust (PASSAT) and 2011 IEEE Third Inernational Conference on Social Computing (SocialCom), 2011 IEEE Third International Conference on (pp. 728-733). IEEE. 